DRAFT VERSION NOVEMBER 6, 2018

Preprint typeset using LATEX style emulateapj v. 08/22/09

\title{
DUST TO DUST: 3 YEARS IN THE EVOLUTION OF THE UNUSUAL SN 2008S
}

\author{
D. M. Szczygiet ${ }^{1}$, J. L. Prieto ${ }^{2,7}$, C. S. KochaneK ${ }^{1,4}$, K. Z. StaneK ${ }^{1,4}$, T. A. Thompson ${ }^{1,4}$, J. F. Beacom ${ }^{1,3,4}$, \\ P. M. GARNAVICH ${ }^{5}$, C. E. WOODWARD ${ }^{6}$ \\ Draft version November 6, 2018
}

\begin{abstract}
We obtained late-time optical and near-IR imaging of SN 2008S with the Large Binocular Telescope (LBT), near-IR data with the Hubble Space Telescope (HST), and mid-IR data with the Spitzer Space Telescope (SST). We find that (1) it is again invisible at optical $(U B V R)$ wavelengths to magnitude limits of approximately $25 \mathrm{mag}$, (2) while detected in the near-IR $(H)$ at approximately $24.8 \mathrm{mag}$, it is fading rapidly, and (3) it is still brighter than the progenitor at 3.6 and $4.5 \mu \mathrm{m}$ in the mid-IR with a slow, steady decline. The IR detections in December 2010 are consistent with dust emission at a blackbody temperature of $T \simeq 640 \mathrm{~K}$ and a total luminosity of $L \simeq 200000 L_{\odot}$, much higher than the $L \simeq 40000 L_{\odot}$ luminosity of the obscured progenitor star. The local environment also shows no evidence for massive $\left(M \gtrsim 10 M_{\odot}\right)$ stars in the vicinity of the transient, consistent with the progenitor being a massive AGB star.
\end{abstract}

Subject headings: stars: evolution - stars: supergiants - supernovae:individual (SN 2008S)

\section{INTRODUCTION}

SN 2008S is one of the most mysterious optical transients created by a massive star in the last decade. It was discovered in February 2008 by Arbour \& Boles (2008) in the prolific supernova factory NGC 6946. It was initially classified as a likely "supernova impostor" due to its faint absolute peak magnitude $\left(M_{V} \sim-13 \mathrm{mag}\right)$ and optical spectra dominated by narrow Balmer, $\mathrm{Ca}$ II triplet, and [Ca II] lines in emission (Stanishev et al. 2008; Steele et al. 2008). NGC 6946 had been observed by the Large Binocular Telescope (LBT) the previous year, and the key piece of evidence from these observations was that there was no optical progenitor (Prieto et al. 2008), which was surprising since the "supernova impostors" are believed to be eruptions from very massive $\left(>20-30 M_{\odot}\right)$, evolved stars (e.g., Smith et al. 2010 and references therein) that should have been easily visible in the LBT observations.

The only means of having an optical eruption from a massive star and an invisible progenitor is for the star to be selfobscured by dust that is largely destroyed by the transient. This possibility was confirmed when Prieto et al. (2008) found the progenitor star as a $\log L / L_{\odot} \simeq 4.5, T \simeq 440 \mathrm{~K}$ blackbody in archival Spitzer data. This luminosity is comparable to that of an evolved $\sim 10 M_{\odot}$ star, and is well below that corresponding to the more massive stars thought to be required for non-supernova eruptions. Subsequent analyses of the progenitor by Botticella et al. (2009) and Wesson et al. (2010) were consistent with those by Prieto et al. (2008).

More remarkably, an almost identical event then occurred in NGC 300 (Monard 2008). The progenitor was invisible in

\footnotetext{
${ }^{1}$ Department of Astronomy, The Ohio State University, 140 W. 18th Ave., Columbus OH 43210

${ }^{2}$ Department of Astrophysical Sciences, Princeton University, Peyton Hall, Princeton, NJ 08544

${ }^{3}$ Department of Physics, The Ohio State University, 191 W. Woodruff Ave., Columbus OH 43210

${ }^{4}$ Center for Cosmology and AstroParticle Physics, The Ohio State University, $191 \mathrm{~W}$. Woodruff Ave., Columbus OH 43210

${ }^{5}$ University of Notre Dame, 225 Nieuwland Science Hall, Notre Dame, IN 46556

6 Minnesota Institute for Astrophysics, School of Physics and Astronomy, 116 Church Street, S. E., University of Minnesota, Minneapolis, MN 55455

${ }^{7}$ Hubble and Carnegie-Princeton Fellow
}

the optical to even tighter limits (Berger \& Soderberg 2008; Bond et al. 2009; Berger et al. 2009), but we again found the progenitor as a self-obscured star of similar luminosity and (dust photosphere) temperature in Spitzer mid-IR data (Prieto 2008; Thompson et al. 2009). A subsequent analysis of the progenitor by Berger et al. (2009) agreed with our estimates, and an investigation of the progenitor based on its neighboring stars by Gogarten et al. (2009) was consistent with the progenitor being a massive star of order $10-20 M_{\odot}$, where the analysis favored the upper portions of this range but, strictly speaking, the method only provides an upper mass bound.

In Thompson et al. (2009) we surveyed the galaxy M33 for mid-IR sources with similar properties to these progenitors and found that they were astonishingly rare, with only a few such sources in the entire galaxy. In the mid-IR, these sources have the properties of super-AGB stars, with properties distinct from other classes of massive stars such as LBVs and red supergiants. The rarity of these sources compared to all massive stars, confirmed in our survey of additional galaxies (Khan et al. 2010), means that the progenitors of the transients are a very short lived $\left(\sim 10^{4}\right.$ years $)$ phase in the evolution of these massive stars and that there is a causal connection between obscuration and explosion.

Thompson et al. (2009) concluded that there are a number of possible mechanisms to explain the nature of these transients and their progenitors: (1) massive white-dwarf birth; (2) electron-capture supernova; (3) intrinsically lowluminosity iron core-collapse supernova; and (4) massive star outbursts. Debates about these possible origins have been raging ever since then, based both on theoretical and observational arguments. They are basically divided into the (some kind of) supernova camp (Prieto et al. 2008; Botticella et al. 2009; Pumo et al. 2009) and the (some kind of) massive star outburst camp (Berger et al. 2009; Smith et al. 2009; Bond et al. 2009; Kashi et al. 2010; Humphreys et al. 2011). The outburst camp generally argues that the progenitor was not $\mathrm{a} \sim 10 M_{\odot}$ super-AGB star but a more massive $15-20 M_{\odot}$ star (supported by Gogarten et al. 2009), despite their position at the red, high luminosity end of the AGB sequence in mid-IR color-magnitude diagrams (Thompson et al. 2009; Khan et al. 2010) and the low mass compared to typical stars 


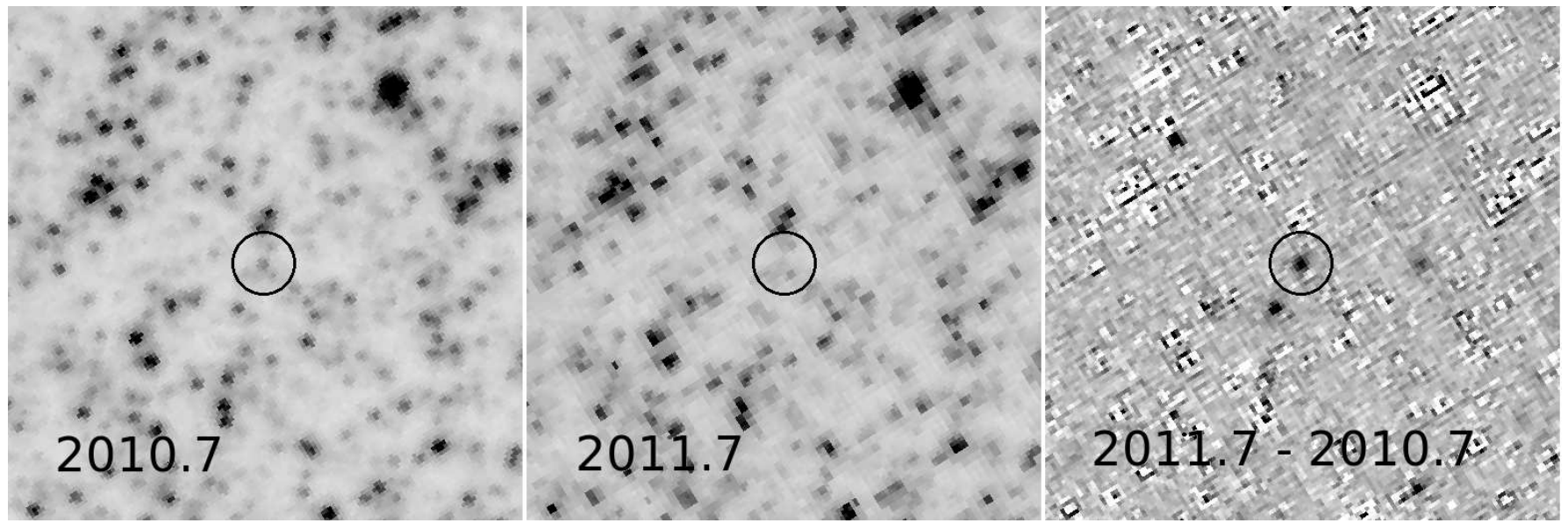

FIG. 1.- The HST F160W $(H)$ band observations of SN 2008S from August 2010 (left) and 2011 (middle). The right panel shows the difference between the 2011 and 2010 epochs, where black means that the source has become fainter. There are other variables in the field of view, but with only two epochs we cannot

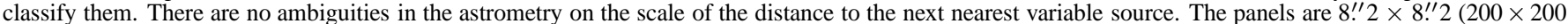
pc) and the radius of the circle is $0 .{ }^{\prime \prime} 5$.

TABLE 1

PHOTOMETRY OF SN 2008S

\begin{tabular}{|c|c|c|c|c|c|c|c|c|c|c|}
\hline $\begin{array}{l}\text { Date } \\
\text { (UT) }\end{array}$ & MJD & $\begin{array}{c}U s \\
(\mathrm{mag})\end{array}$ & $\begin{array}{c}B \\
(\mathrm{mag})\end{array}$ & $\begin{array}{c}V \\
\text { (mag) }\end{array}$ & $\begin{array}{c}R \\
(\mathrm{mag})\end{array}$ & $\begin{array}{c}J \\
(\mathrm{mag})\end{array}$ & $\begin{array}{c}H \\
(\mathrm{mag})\end{array}$ & $\begin{array}{c}K \\
(\mathrm{mag})\end{array}$ & $\begin{array}{c}{[3.6]} \\
\text { (mag) }\end{array}$ & $\begin{array}{c}{[4.5]} \\
(\mathrm{mag})\end{array}$ \\
\hline $2008-02-06$ & 54503.3 & & & & $\cdots$ & $\cdots$ & $\cdots$ & $\cdots$ & $13.11 \pm 0.01$ & $12.40 \pm 0.01$ \\
\hline $2008-05-03$ & 54589.4 & $21.49 \pm 0.07$ & $20.86 \pm 0.03$ & $19.46 \pm 0.04$ & $18.47 \pm 0.03$ & $\ldots$ & $\ldots$ & $\ldots$ & $\ldots$ & $\ldots$ \\
\hline $2008-05-04$ & 54590.4 & $21.52 \pm 0.08$ & $20.91 \pm 0.03$ & & $18.48 \pm 0.03$ & $\ldots$ & $\ldots$ & $\ldots$ & $\ldots$ & $\ldots$ \\
\hline $2008-07-05$ & 54652.4 & $22.72 \pm 0.07$ & $22.27 \pm 0.03$ & $21.16 \pm 0.04$ & $20.03 \pm 0.04$ & $\ldots$ & $\ldots$ & $\ldots$ & $\ldots$ & $\ldots$ \\
\hline 2008-07-18 & 54666.2 & $\ldots$ & & & $\ldots$ & $\ldots$ & $\ldots$ & $\ldots$ & $14.97 \pm 0.01$ & $14.04 \pm 0.01$ \\
\hline $2008-11-22$ & 54792.1 & $\ldots$ & $23.59 \pm 0.05$ & $22.50 \pm 0.05$ & $\ldots$ & $\ldots$ & $\ldots$ & $\ldots$ & $\ldots$ & $\ldots$ \\
\hline $2008-11-23$ & 54793.1 & $\ldots$ & $23.58 \pm 0.06$ & $22.56 \pm 0.05$ & $\ldots$ & $\ldots$ & $\ldots$ & $\ldots$ & $\ldots$ & $\ldots$ \\
\hline $2008-11-24$ & 54794.1 & $\ldots$ & $23.45 \pm 0.05$ & $22.45 \pm 0.05$ & $\cdots$ & $\ldots$ & $\ldots$ & $\ldots$ & $\ldots$ & $\cdots$ \\
\hline $2008-11-25$ & 54795.1 & $\ldots$ & $23.54 \pm 0.06$ & $22.60 \pm 0.05$ & $\ldots$ & $\ldots$ & $\ldots$ & $\ldots$ & $\ldots$ & $\ldots$ \\
\hline $2009-03-25$ & 54915.5 & $<24.1$ & $<25.6$ & $<24.8$ & $23.10 \pm 0.07$ & $\ldots$ & $\ldots$ & $\ldots$ & $\ldots$ & $\ldots$ \\
\hline $2009-10-20$ & 55124.1 & $<25.2$ & $<25.9$ & $<25.7$ & $<25.1$ & $\ldots$ & $\ldots$ & $\ldots$ & $\ldots$ & $\ldots$ \\
\hline $2009-10-22$ & 55126.1 & $<24.9$ & $<25.9$ & $<25.6$ & $<25.1$ & $\ldots$ & $\ldots$ & $\ldots$ & $\ldots$ & $\ldots$ \\
\hline $2009-12-17$ & 55182.0 & $\ldots$ & $\ldots$ & $\ldots$ & $\ldots$ & $\ldots$ & $20.31 \pm 0.14$ & $\ldots$ & $\ldots$ & $\ldots$ \\
\hline 2010-03-17 & 55272.5 & & 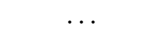 & & 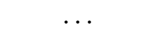 & $\ldots$ & $<21.4$ & $19.23 \pm 0.09$ & $\ldots$ & $\ldots$ \\
\hline 2010-03-18 & 55237.5 & $<24.6$ & $<25.3$ & $<25.4$ & $<24.9$ & $\ldots$ & $\ldots$ & & $\ldots$ & $\ldots$ \\
\hline $2010-05-17$ & 55333.4 & $\ldots$ & $\ldots$ & $\ldots$ & $\ldots$ & $\ldots$ & $\ldots$ & $20.27 \pm 0.15$ & $\ldots$ & $\ldots$ \\
\hline $2010-08-08$ & 55417.3 & $\ldots$ & $\ldots$ & $\ldots$ & $\ldots$ & $\ldots$ & $\ldots$ & $\ldots$ & $15.91 \pm 0.02$ & $14.66 \pm 0.01$ \\
\hline $2010-08-24$ & 55433.2 & $\ldots$ & $\ldots$ & $\ldots$ & $\ldots$ & $<25.9$ & $23.00 \pm 0.03$ & $\ldots$ & & \\
\hline 2010-12-01 & 55531.9 & $\ldots$ & $\ldots$ & $\ldots$ & $\ldots$ & $\ldots$ & ... & $\ldots$ & $16.16 \pm 0.02$ & $14.86 \pm 0.01$ \\
\hline 2011-08-07 & 55780.6 & $\ldots$ & $\ldots$ & $\ldots$ & $\ldots$ & $<26.5$ & $24.78 \pm 0.21$ & $\ldots$ & $\ldots$ & $\ldots$ \\
\hline
\end{tabular}

All the magnitude upper limits are $3 \sigma$. The estimated start date of the transient is MJD 54485.5 \pm 4 (Botticella et al. 2009). $B, V$ and $R$ are Bessel filters, $U s$ is a high throughput $U$-band interference filter.

with LBV outbursts (see Smith et al. 2010). The massivestar outburst interpretation is seriously called into question by our Spitzer IRS spectrum of the NGC 300 event (Prieto et al. 2009, also Kwok 2011). The mid-IR spectrum resembles that of carbon-rich proto-planetary nebulae and lacks the silicatedominated dust features typical of massive star outbursts (e.g., Humphreys et al. 2006). Wesson et al. (2010), analyzing postevent Spitzer observations of SN 2008S, also found that the silicate dust characteristics of high mass stars were inconsistent with the observations. Prieto et al. (2009) also note that proto-planetary nebulae (initial masses $\lesssim 8 M_{\odot}$ ) have most of the optical spectral features that led Smith et al. (2009), Bond et al. (2009) and Berger et al. (2009) to argue for an outburst from a more massive $\left(\sim 20 M_{\odot}\right)$ star. Since "Type IIn" optical spectroscopic properties are seen in some proto-planetary nebulae, massive supergiants, supernova impostors, and the genuine, but very diverse, Type IIn supernovae, they appear only to be a diagnostic for the presence of strong interactions between ejecta and a dense circumstellar medium rather than a diagnostic for the source of the ejecta.

Most recently Kochanek (2011) re-analyzed the available data for SN 2008S and NGC 300-OT and concluded that both transients were of explosive nature. If the transients were not explosive, the energy would not be sufficient to destroy the obscuring dust and make the transients visible in the optical. After the peak of the transient, the dust rapidly reforms from the outside, and the luminosity, which then comes from the shock propagating through the dense wind, is mostly reradiated by that dust in the IR. The model predicts that the peak obscuration is 2-3 years post-transient, when the shock reaches the reformed dust shell and pushes its radius outwards. It should take another 5-10 years (in the case of SN 2008S) before it is possible to see through the dust and determine the fate of the progenitor star. 


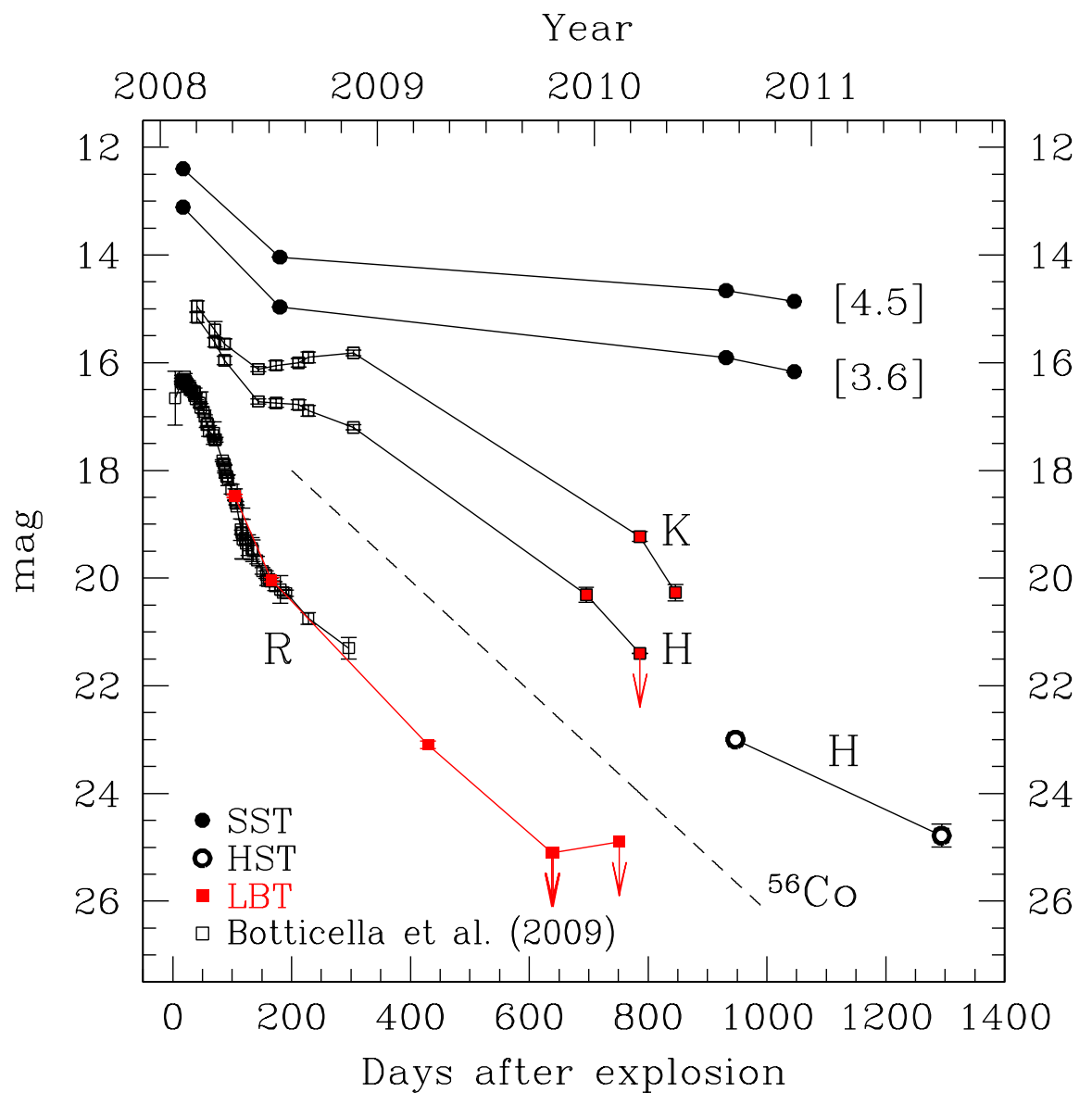

FIG. 2.- The $R, H$ and $K$-band light curves of SN 2008S from Botticella et al. (2009, open black points), the Large Binocular Telescope (filled red points) and HST (open circles). Filled circles show the 3.6 and $4.5 \mu \mathrm{m}$ Spitzer light curves. Upper limits are indicated by arrows. Botticella et al. (2009) noted that the early ( $<200$ days) bolometric light curve had a slope consistent with that for ${ }^{56} \mathrm{Co}$ decay (indicated by the dashed line), but this is clearly inconsistent with the late time light curve.

Here we report the data on SN 2008S used in this analysis. We have been following the SN 2008S event with the LBT in both the optical and near-IR, with HST in the near-IR, and with SST in the mid-IR. Here we report that the source is again too faint to detect in the optical, marginally detected in near-IR HST images (see Fig. 1), and still bright in the midIR. It is presently much more luminous than the progenitor and slowly fading. We describe our observations and results in $\S 2$ and discuss their implications in $\S 3$.

\section{OBSERVATIONS AND RESULTS}

The optical observations were done with the Large Binocular Cameras (LBC, Giallongo et al. 2008), using the LBC/Blue camera for $U, B$ and $V$ and the LBC/Red camera for $R$. The pixel scale of the LBC cameras is $0 . / 224$. Since these observations are part of a program whose overall goal is to use difference imaging to characterize variable sources, the sub-images obtained for each epoch were not dithered and SN 2008S was always located at approximately the same point on Chip 2 of the cameras. Image exposure times were $300 \mathrm{sec}$, generally with two exposures for $U, B$ and $V$ and 6 exposures for $R$. The near-IR observations were made with LUCIFER (Seifert et al. 2003; Mandel et al. 2008; Ageorges et al. 2010) in the $H$ and $K$ bands using the F3.75 camera with a pixel scale of $0 . \prime 12$. At each dither position we obtained 3 exposures of 33 (10) sec for $H(K)$ band. We obtained 10 on- source and 6 off-source dither positions in a 2-5-2-5-2 off-onoff-on-off pattern, where the off-source position was shifted 8 arcmin away from the galaxy. Another set of near-IR observations was obtained with the Hubble Space Telescope (HST) WFC3/IR camera in $F 110 W(J)$ and $F 160 W(H)$ filters (proposal ID 12331). The exposure times were $1 x 800$ s in $J$ and $3 \times 700$ s in $H$ and were taken in August 2010 and 2011. In addition to the near-IR HST observations, we obtained mid-IR data with warm Spitzer on August and December 2010 (program ID 70040), with exposure times of $8 \times 30$ s at both [3.6] and [4.5].

The optical and near-IR LBT data were reduced using standard methods in IRAF. The photometry was obtained using DAOPHOT and ALLSTAR (Stetson 1987; Stetson 1992). The optical data was calibrated using 4-24 local standards from Welch et al. (2007) for the $V$ and $R$ bands and from Botticella et al. (2009) for the $U$ and $B$ bands. The nearIR LBT data were calibrated using 3-6 2MASS stars in the field. In both cases we only applied a zero-point offset to convert the instrumental magnitudes into the standard system. The photometry on the HST near-IR data was made with the DOLPHOT package (Dolphin 2000) utilizing the WFC3 module which provides specific PSFs and pixel area maps and automatically applies all the necessary corrections. For the mid-IR SST data, we used aperture photometry with an aperture radius 4 pixels (2." 4 ) and a background annulus 4-12 pix- 


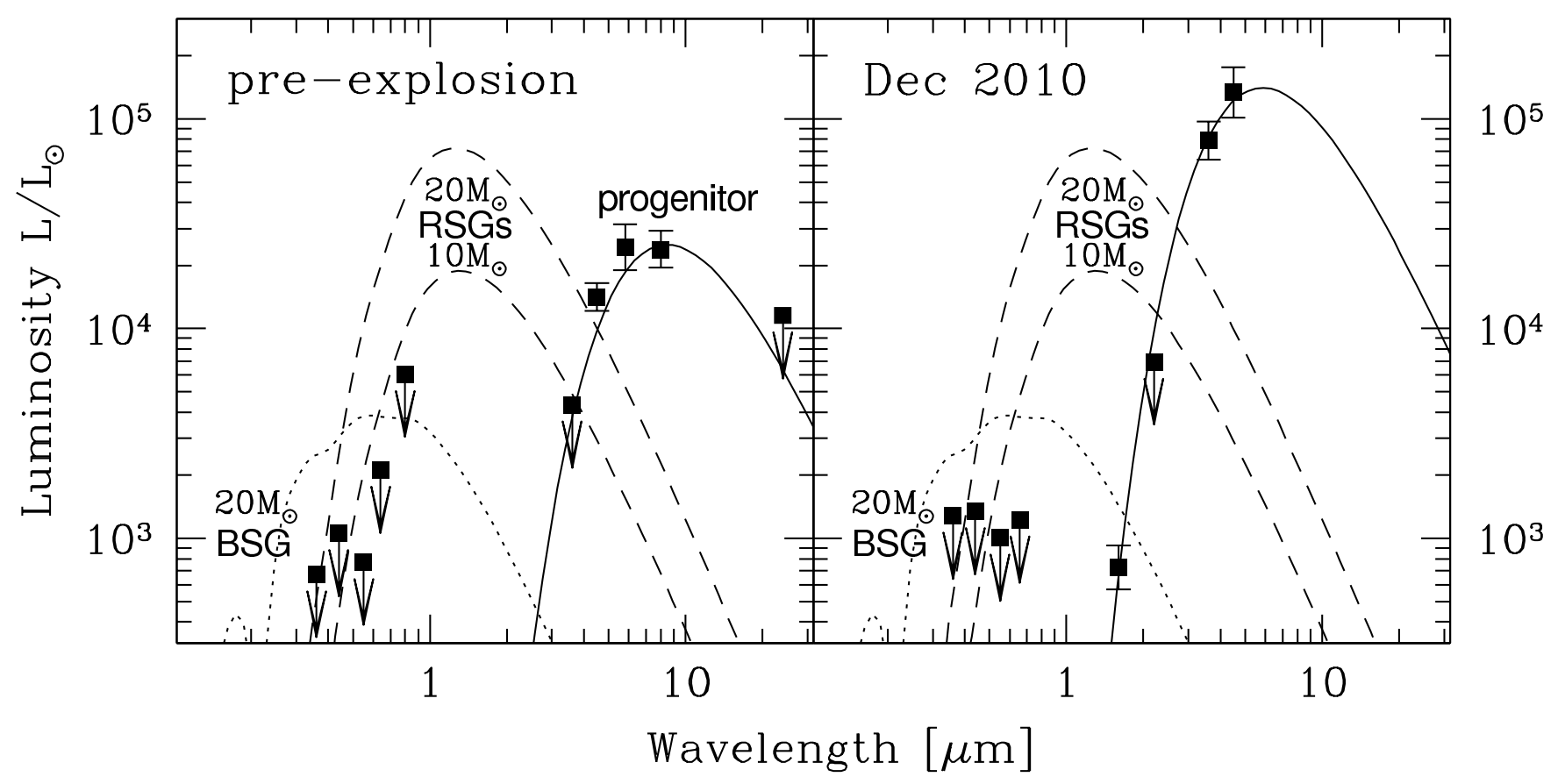

FIG. 3.- The pre-explosion, progenitor SED (left) and the current SED (right) of SN 2008S as of December 2010. The measured magnitudes are converted to fluxes, and these are converted to a luminosity as $L=4 \pi D^{2} \nu F_{\nu}$ where $D=5.6 \mathrm{Mpc}$. The SED models are just blackbodies plus $A_{V}=2.13$ mag of total extinction. The $10 M_{\odot}$ and $20 M_{\odot}$ red supergiant models (RSG, dashed curves) are from Marigo et al. (2008) and have $T \simeq 3600$ and $3900 \mathrm{~K}$ with $\log L / L \odot=4.68$ and 5.29 , respectively. The blue supergiant model (dotted curve) is based on SN1987A and has $T \simeq 16000 \mathrm{~K}$ and $\log L / L_{\odot}=5.0$. The best fit blackbody model (solid curve) for the progenitor has $T=440 \mathrm{~K}$ and $\log L / L_{\odot}=4.54$ (Prieto et al. 2008) while the December 2010 model is consistent with $T=640 \mathrm{~K}$ and $\log L / L_{\odot}=5.30$. The last available LBT $K$-band observation (see Table 1) is used as an upper limit on the right panel.

els. We applied the aperture corrections of 1.213 for [3.6] and 1.234 for [4.5] $\mu \mathrm{m}$ provided in the SST IRAC Instrument Handbook. ${ }^{8}$ The multi-wavelength results are presented in Table 1, where the magnitude errors include the uncertainties both in the measurements and in the zero points. In the cases where we do not detect SN 2008S, we place a $3 \sigma$ upper limit on the magnitude using the standard deviation of the sky in a region around the source.

Figure 2 shows the $H, K$ and $R$-band light curves from Botticella et al. (2009) and our LBT observations, as well as the $H$-band HST and [3.6]/[4.5] $\mu \mathrm{m}$ SST data points. The SN is not detected in the HST $J$-band and we do not plot the limits for clarity. For the same reason we do not plot the most recent $R$-band LBT limits. Figure 3 shows the progenitor and current (as of December 2010) spectral energy distributions (SED). The left panel of Fig. 3 shows the constraints on the progenitor's SED as compared to typical massive stars. To make the comparison we used a Galactic plus intrinsic extinction of $A_{V}=2.13 \mathrm{mag}$ (Botticella et al. 2009) and the distance of $D=5.6 \mathrm{Mpc}$ adopted by Prieto et al. (2008). The data points are converted to a luminosity as $L=4 \pi D^{2} \nu F_{\nu}$. For comparison we show the extincted SEDs of $10 M_{\odot}$ and $20 M_{\odot}$ red supergiants (RSG) using luminosities and effective temperatures from Marigo et al. (2008), a $20 M_{\odot}$ blue supergiant (BSG) modeled on SN1987A, and the blackbody that best fit the SN 2008S progenitor data (solid line).

In the optical $(U B V R)$, the source is again too faint to correspond to a massive $\left(>10 M_{\odot}\right)$ evolved star, with limits on its brightness similar to those for the progenitor (see right panel in Fig. 3). The extinction would have to be increased from the
$A_{V} \simeq 2.1$ mag estimated to be present post-explosion (Botticella et al. 2009) to $A_{V} \sim 3.6-5.8 \mathrm{mag}$ in order to obscure the models shown in Fig. 3. The transient is now marginally detectable in the HST near-IR (see the middle panel of Fig. 1), and it is fading with a slope of $1.9 \pm 0.25 \mathrm{mag} / \mathrm{year}$ between the 2 HST epochs which is less steep than the mean slope of $2.9 \pm 0.2 \mathrm{mag} / \mathrm{year}$ between the late phases of the Botticella et al. (2009) $H$-band light curve and our first LBT observation. The decline at $K$-band between the last two LBT epochs is very rapid, approximately $6 \pm 1 \mathrm{mag} / \mathrm{year}$ and is significantly steeper than the mean slope of $2.3 \pm 0.1 \mathrm{mag} / \mathrm{year}$ between the Botticella et al. (2009) and LBT light curves. The SED is rising to the red with $H-K>2.2$ mag 800 days after explosion. If we extrapolate the $H$-band flux from December 2009 to March 2010 (which is the last $K$-band data point) by connecting the last LBT and first HST detections, we estimate $H \simeq 21.9$ mag and thus $H-K \simeq 2.7 \mathrm{mag}$, which is significantly redder than the $H-K \simeq 1.4$ mag color in the late phases of Botticella et al. (2009).

We can roughly estimate a temperature and luminosity for the March 2010 epoch. Fitting a blackbody to the measured $K$-band flux and the extrapolated $H$-band estimate $(21.9 \mathrm{mag})$, we get a temperature of $700 \mathrm{~K}$ and a luminosity of $280000 L_{\odot}$. When we estimate a temperature and luminosity for the last epoch of SST observations (December 2010) we get $T=$ $640 \mathrm{~K}$ and $L=200000 L_{\odot}$. In the DUSTY (Ivezić \& Elitzur 1997) models of these data by Kochanek (2011), the estimated luminosity at this epoch is $\log L / L_{\odot}=5.6 \pm 0.6$. Even with the further fading between March and December 2010, the source luminosity is still much higher than the estimated luminosity $L \simeq 40000 L_{\odot}$ of the progenitor star (Prieto et al. 2008; Bot-

\footnotetext{
${ }^{8}$ http://irsa.ipac.caltech.edu/data/SPITZER/docs/irac/iracinstrumenthandbook/28ticella et al. 2009; Wesson et al. 2010). The source has faded
} 
by another $1.25 \mathrm{mag}$ in the $H$-band between December 2010 and August 2011 and by now is probably undetectable in the near-IR. Future warm Spitzer observations will allow us to follow the mid-IR evolution of the light curve, but without cold Spitzer data the SED will be hard to constrain.

Fig. 4 shows near-IR $H / J-H$ color magnitude diagrams (CMD) for both a large $(2.3 \times 2.0$ arcmin $)$ and a small 4 ." $1^{\prime \prime}$ $(100 \mathrm{pc})$ radius region around the source (this smaller region is shown in Fig. 1). These assume a distance of 5.6 Mpc, following Prieto et al. (2008), and are corrected for $E(B-V)=$ $0.342 \mathrm{mag}$ of foreground Galactic extinction (Schlegel et al. 1998). To construct the CMDs we combined the 2010 and 2011 HST epochs for each band and then used DOLPHOT to perform photometry on the combined images. The resulting photometric catalogs contained 82,000 sources. Following Dalcanton et al. (2011), we then required the signal-tonoise ratio in both filters to be greater than 4 for a detection, and a sharpness parameter sharpness ${ }^{2}<0.1$ to exclude nonstellar sources from the catalog, which left 67,000 sources. The resulting CMD is dominated by old ( $\gtrsim 1 \mathrm{Gyr}$ ) stellar populations (see Fig. 10 from Dalcanton et al. (2011)). The red giant branch $(\mathrm{RGB})$ is visible at $F 110 W-F 160 W \sim 1.0$, but the red clump (RC), usually found 3-4 magnitudes below the tip of the RGB (at $M_{H} \simeq-6$ ), is too faint to be resolved in our data, mainly because of the high crowding. The location of SN 2008S is marked with a circle for the 2010 epoch and with a square for the 2011 epoch. It is very faint and red compared to other stars in the field.

When we examine the CMD of the stars within a 4 !" $1^{\prime \prime}$ (100 pc) radius of SN $2008 \mathrm{~S}$ in the middle panel of Fig. 4, we see very few luminous stars. The superposed Padova (Marigo et al. 2008) isochrones for $10^{7}, 10^{7.3}, 10^{7.5}$ and $10^{8}$ years have ZAMS masses corresponding to their end points of 19, 13, 9 and $5 M_{\odot}$, respectively. Unless the local extinction is high, as indicated by the arrow corresponding to the effect of $A_{V}=3$ of additional extinction, the region lacks the population of young stars that should be associated with a high mass $\left(M \gtrsim 10 M_{\odot}\right)$ progenitor for SN 2008S, but does show evidence for a population with $M \simeq 10 M_{\odot}$. For contrast, the right panel of Fig. 4 shows the CMD for a $4 !^{\prime \prime} 1(100 \mathrm{pc})$ radius region around the Type IIP SN 2002hh (Pozzo et al. 2006). Here we see a population of massive stars extending up to the $10^{7} \mathrm{yrs}$ isochrone, corresponding to a maximum mass of $19 M_{\odot}$, which is wellmatched to the upper mass limit of $M<18 M_{\odot}$ for the progenitor of SN 2002hh by Smartt et al. (2009).

\section{DISCUSSION}

Thompson et al. (2009) proposed that SN 2008S and the NGC 300 transient were the archetypes of a new class of transients potentially including the M85 OT-1 transient (Kulkarni et al. 2007; Pastorello et al. 2007), SN 1999bw (Li et al. 2002 and references therein), and now PTF10fqs (Kasliwal et al. 2010). The initial defining characteristics were (1) a dust-enshrouded progenitor without optical counterpart and mid-IR magnitudes that places them at the tip of the AGB sequence in a mid-IR CMD, and (2) a low-luminosity transient $\left(-13 \gtrsim M_{V} \gtrsim-15\right)$ with narrow lines in emission in the spectra $(v \lesssim 3000 \mathrm{~km} / \mathrm{s})$, and signs of a circumstellar dust excess at near-IR and mid-IR wavelengths. Examinations of the dust properties (Prieto et al. 2009; Wesson et al. 2010) suggest (3) that the dust is carbonaceous rather than the silicate dust seen in massive stars.

Here we add (4) that the progenitor either does not survive or must return to its dust enshrouded state. As the right panel of Fig. 3 shows, the LBT data already rule out the presence of a massive, evolved star unless it has reconstituted an optically thick, dusty envelope. The present optical limits are somewhat stronger than those for the progenitor, and the near-IR detections already rule out RSGs more massive than $10 M_{\odot}$ unless more heavily obscured. The total luminosity is still much higher than that of the progenitor and emerges mainly in the mid-IR, but it is also slowly fading. Prieto et al. (2010) and Ohsawa et al. (2010) find that the NGC 300 transient has also vanished in the optical but remains bright in the mid-IR.

In the Kochanek (2011) scenario, the shock powering the present luminosity should eventually destroy the dust, and we will be able to observe the direct emission from the shock and any surviving star. This will likely require, however, monitoring these sources for almost a decade. These observations, and the similar data for the NGC 300 transient, demonstrate, however, that it is impossible to understand these transients based only on optical observations - understanding their evolution, luminosities, and energetics requires mid-IR observations.

The observational properties of the progenitors are broadly consistent with being massive $\left(M \simeq 10 M_{\odot}\right)$ AGB stars. The one significant counterargument has been the observation by Gogarten et al. (2009) that the stellar populations near the NGC 300 transient are consistent with a progenitor mass as high as $\sim 20 M_{\odot}$. This approach technically only yields an upper bound on the mass - allowing, but not requiring, the NGC 300 progenitor to be significantly more massive than any AGB star. For the neighborhood of SN 2008S, we see no evidence for a population of stars significantly more massive than $\sim 10 M_{\odot}$ unless there is an enormous amount of (uniform) local extinction. This is based, however, on a near-IR CMD in which it is difficult to constrain extinctions and temperatures. Adding optical observations would make it clear whether there is any possibility of younger more massive stars in the region.

We thank G. Cresci, J. Hill, R. Humphreys, and A. Quirrenbach for suggestions and comments. JLP acknowledges support from NASA through Hubble Fellowship grant HF51261.01-A awarded by STScI, which is operated by AURA, Inc. for NASA, under contract NAS 5-2655. CSK, JLP, KZS, DS and TAT are supported in part by NSF grant AST0908816. JFB is supported by NSF CAREER Grant PHY0547102 and NSF Grant PHY-1101216. Based in part on observations made with the Large Binocular Telescope. The LBT is an international collaboration among institutions in the United States, Italy and Germany. The LBT Corporation partners are: the University of Arizona on behalf of the Arizona university system; the Istituto Nazionale di Astrofisica, Italy; the LBT Beteiligungsgesellschaft, Germany, representing the Max Planck Society, the Astrophysical Institute Potsdam, and Heidelberg University; the Ohio State University; and the Research Corporation, on behalf of the University of Notre Dame, University of Minnesota and University of Virginia. This work is based in part on observations made with the Spitzer Space Telescope, which is operated by the Jet Propulsion Laboratory, California Institute of Technology under a contract with NASA. Support for this work was provided by NASA through award 1414623 issued by JPL/Caltech. Support for HST program GO-12331 was provided by NASA through a grant from the Space Telescope Science Institute, which is operated by the Association of Uni- 


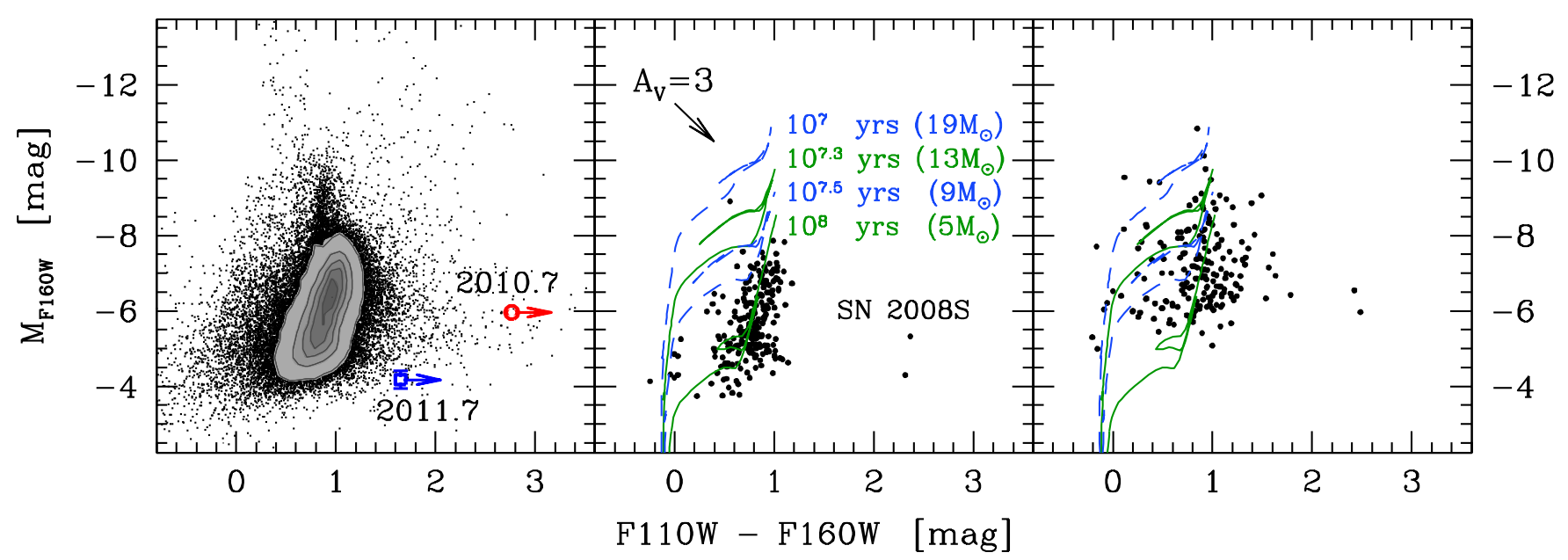

FIG. 4.- Near-IR CMDs from the combined HST epochs. The left panel shows a large region $(2.3 \times 2.0$ arcmin $)$ while the middle and right panels show a 4 .' 1 $(100 \mathrm{pc})$ radius around SN 2008S and SN 2002hh, respectively. The CMDs are corrected for Galactic extinction and assume a distance of $5.6 \mathrm{Mpc}$. The location of SN 2008S is marked with a circle for the 2010 epoch and with a square for the 2011 epoch. The curves on the middle and right panels show Padova (Marigo et al. 2008) isochrones for $10^{7}, 10^{7.3}, 10^{7.5}$ and $10^{8}$ years, alternating solid and dashed lines, which have end points corresponding to ZAMS masses of $19,13,9$ and $5 M_{\odot}$ respectively. The top of the two reddest points in the middle panel is the epoch-averaged measurement for SN $2008 \mathrm{~S}$.

versities for Research in Astronomy, Inc., under NASA con-

Facilities: LBT, HST, SST tract NAS5-26555.

\section{REFERENCES}

Ageorges, N., et al., 2010, Proc. SPIE, 7735, to be published

Arbour, R., \& Boles, T. 2008, Central Bureau Electronic Telegrams, 1234, 1

Berger, E., \& Soderberg, A. 2008, The Astronomer's Telegram, 1544, 1

Berger, E., et al. 2009, ApJ, 699, 1850

Bond, H. E., Bedin, L. R., Bonanos, A. Z., Humphreys, R. M., Monard,

L. A. G. B., Prieto, J. L., \& Walter, F. M. 2009, ApJ, 695, L154

Botticella, M. T., et al. 2009, MNRAS, 398, 1041

Dalcanton, J. J., et al. 2012, ApJS, 198, 6

Draine, B. T. 1981, ApJ, 245, 880

Dolphin, A. E. 2000, PASP, 112, 1383

Giallongo, E., et al. 2008, A\&A, 482, 349

Gogarten, S. M., Dalcanton, J. J., Murphy, J. W., Williams, B. F., Gilbert, K., \& Dolphin, A. 2009, ApJ, 703, 300

Humphreys, R. M., et al. 2006, AJ, 131, 2105

Humphreys, R. M., Bond, H. E., Bedin, L. R., Bonanos, A. Z., Davidson, K., Monard, B. L. A. G., Prieto, J. L., Walter, F. M. 2011, ApJ, 743, 118

Ivezić, Ž., \& Elitzur, M. 1997, MNRAS, 287, 799

Kashi, A., Frankowski, A., \& Soker, N. 2010, ApJ, 709, L11

Kasliwal, M. M., et al. 2011, ApJ, 730, 134

Khan, R., Stanek, K. Z., Prieto, J. L., Kochanek, C. S., Thompson, T. A., \& Beacom, J. F. 2010, ApJ, 715, 1094

Kochanek, C. S. 2011, ApJ, 741, 37

Kulkarni, S. R., et al. 2007, Nature, 447, 458

Kwok, S. 2011, IAU Symposium, 280, 203

Li, W., Filippenko, A. V., Van Dyk, S. D., Hu, J., Qiu, Y., Modjaz, M., \& Leonard, D. C. 2002, PASP, 114, 403

Mandel, H., et al. 2008, Proc. SPIE, 7014

Marigo, P., Girardi, L., Bressan, A., Groenewegen, M. A. T., Silva, L., \& Granato, G. L. 2008, A\&A, 482, 883

Monard, L. A. G. 2008, IAU Circ., 8946, 1
Ohsawa, R., et al. 2010, ApJ, 718, 1456

Pastorello, A., et al. 2007, Nature, 449, 1

Pozzo, M., Meikle, W. P. S., Rayner, J. T., et al. 2006, MNRAS, 368, 1169 Prieto, J. L. 2008, The Astronomer's Telegram, 1550, 1

Prieto, J. L., et al. 2008, ApJ, 681, L9

Prieto, J. L., Sellgren, K., Thompson, T. A., \& Kochanek, C. S. 2009, ApJ, 705,1425

Prieto, J. L., et al. 2010, The Astronomer's Telegram, 2406, 1

Pumo, M. L., et al. 2009, ApJ, 705, L138

Schlegel, D. J., Finkbeiner, D. P., \& Davis, M. 1998, ApJ, 500, 525

Seifert, W., et al. 2003, Proc. SPIE, 4841, 962

Smartt, S. J., Eldridge, J. J., Crockett, R. M., Maund, J. R. 2009, MNRAS, 395, 1409

Smith, N., et al. 2009, ApJ, 697, L49

Smith, N., et al. 2010, AJ, 139, 1451

Stanishev, V., Pastorello, A., \& Pursimo, T. 2008, Central Bureau Electronic Telegrams, 1235,1

Steele, T. N., Silverman, J. M., Ganeshalingam, M., Lee, N., Li, W., \&

Filippenko, A. V. 2008, Central Bureau Electronic Telegrams, 1275, 1

Stetson, P. B. 1987, PASP, 99, 191

Stetson, P. B. 1992, Astronomical Data Analysis Software and Systems I, 25, 297

Thompson, T. A., Prieto, J. L., Stanek, K. Z., Kistler, M. D., Beacom, J. F., \& Kochanek, C. S. 2009, ApJ, 705, 1364

Welch, D. L., Clayton, G. C., Campbell, A., Barlow, M. J., Sugerman,

B. E. K., Meixner, M., \& Bank, S. H. R. 2007, ApJ, 669, 525

Wesson, R., et al. 2010, MNRAS, 403, 474 